\title{
The Impact of Monetary Reward on Memory in Schizophrenia Spectrum Disorder
}

\author{
Allen E. Thornton \\ Simon Fraser University and University of British Columbia \\ Todd S. Woodward \\ Riverview Hospital and Simon Fraser University
}

\author{
Vanessa G. Boudreau and Stephanie Y. Griffiths \\ Simon Fraser University
}

Tanya Fawkes-Kirby

Simon Fraser University

William G. Honer

University of British Columbia

\begin{abstract}
The impact of monetary reward on verbal working memory (vWM) and verbal long-term memory (vLTM) was evaluated in 50 patients with schizophrenia spectrum disorders and 52 matched healthy participants. This research was motivated by the observations that negative symptoms in schizophrenia are associated with reduced drive and that patients with these symptoms exhibit greater mnemonic impairments. Reward-related gains were evaluated across two levels of vWM load on the $n$-back task and across three aspects of vLTM derived from the California Verbal Learning Test-II (i.e., learning, total immediate recall, and retention). Although healthy individuals benefited from reward at a high $v W M$ load level, schizophrenia patients exhibited no reward-related improvements in vWM. In contrast, improvement in vLTM retention was induced by reward for both patients and controls. Finally, symptomatic and pharmacology treatment factors were associated with reward-related gains in persons with schizophrenia. In conclusion, contingent monetary rewards delivered during vWM and vLTM enhanced specific aspects of memory. The influence was relatively small and dependent on the specific neurocognitive operation examined, the mental health status of the participants, and for patients, their particular symptoms and pharmacological treatments.
\end{abstract}

Keywords: working memory, long-term memory, episodic memory, reward, motivation

Individuals with schizophrenia spectrum disorder exhibit a broad array of neurocognitive deficits (e.g., Heinrichs \& Zakzanis, 1998) with evidence of anomalies even in patients with overall abilities that are above average (Wilk et al., 2005). Influences from

Allen E. Thornton, Department of Psychology, Simon Fraser University, Burnaby, British Columbia, Canada, and Centre for Complex Disorders, Department of Psychiatry, University of British Columbia, Vancouver, British Columbia, Canada; Vanessa G. Boudreau, Stephanie Y. Griffiths, and Tanya Fawkes-Kirby, Department of Psychology, Simon Fraser University, Burnaby, British Columbia, Canada; Todd S. Woodward, Department of Research, Riverview Hospital, Coquitlam, British Columbia, Canada, and Department of Psychology, Simon Fraser University, Burnaby, British Columbia, Canada; William G. Honer, Centre for Complex Disorders, Department of Psychiatry, University of British Columbia, Vancouver, British Columbia, Canada.

This research was supported by a President's Research Grant from Simon Fraser University, a New Emerging Team Grant from the Canadian Institute for Health Research (NET-54013), the British Columbia Mental Health and Addictions Services, and the Michael Smith Foundation for Health Research.

We thank Raymond Koopman for his assistance with portions of the statistical analyses. In addition, we thank Wendy Loken Thornton for her helpful comments on an earlier version of this article. Finally, we thank Hayley Kristinsson and Rachel McKay for their assistance in data collection.

Correspondence concerning this article should be addressed to Allen E. Thornton, Human Neuropsychology Laboratory, Department of Psychology, 8888 University Drive, Simon Fraser University, Burnaby, British Columbia, Canada V5A 1S6. E-mail: aethornt@sfu.ca the reward system might have important implications for the expression of these neurocognitive deficits (Barch, 2005). In healthy individuals, monetary reward influences performance on tasks of working memory and episodic verbal long-term memory (vLTM) and modifies the functional neuroactivations elicited (Lisman \& Grace, 2005; Taylor et al., 2004). Regional neocortical activations during working memory, which involves mesocortical dopamine circuits (Goldman-Rakic, 2004; Robbins, 2005; Tanaka, 2006; Watanabe, Kodama, \& Hikosaka, 1997; Williams \& Castner, 2006; Williams \& Goldman-Rakic, 1995), are generally increased by monetary reward (Potkin et al., 2002; Taylor et al., 2004). This, in turn, modifies working memory performance (Taylor et al., 2004). Additionally, monetary rewards activate midbrain and mesolimbic circuits that facilitate declarative long-term memory (LTM) apparently through dopaminergic influences on the hippocampus (Adcock, Thangavel, Whitfield-Gabrieli, Knutson, \& Gabrieli, 2006; Wittmann et al., 2005; also see Lisman \& Grace, 2005).

The relationship between symptoms, reward, and neurocognition in schizophrenia spectrum disorder is of interest because of the apparent lack of motivation exhibited by many patients, which may reflect aberrant reward processing and subsequent neurocognitive impairments (Barch, 2005). Indeed, motivation deficiencies have been argued to underlie a significant portion of the neurocognitive features of the illness (Carter, 2005). Furthermore, persons with schizophrenia respond to monetary reward cues with comparative attenuation of activation in the ventral striatal reward system, which predicts symptom severity, particularly negative 
manifestations (Juckel, Schlagenhauf, Koslowski, Filonov, et al., 2006; Juckel, Schlagenhauf, Koslowski, \& Wüstenberg, et al., 2006).

More generally, aberrant dopamine transmission may affect motivation, neurocognition, and symptomatology in schizophrenia (Barch, 2005; Nieoullon \& Coquerel, 2003). Indeed, recent formulations of the dopamine hypothesis propose that subcortical dopamine excesses account for positive symptoms of the disorder, whereas cortical dopamine deficiencies result in the negative illness aspects (e.g., Abi-Dargham, 2004; Davis, Kahn, Ko, \& Davidson, 1991; Goldman-Rakic, 2004; Weinberger, 1987). Furthermore, the importance of dopamine in neurocognition is indicated by its role in VLTM and working memory functioning of healthy persons (Backman et al., 2000; Cropley, Fujita, Innis, \& Nathan, 2006; Erixon-Lindroth et al., 2005; Fried et al., 2001; Mozley, Gur, Mozley, \& Gur, 2001; Volkow et al., 1998) and its association with working memory impairments in schizophrenia (AbiDargham et al., 2002; Goldman-Rakic, 2004).

Nonetheless, few studies have focused on the interface between reward, symptomatology, and neurocognition in persons with schizophrenia spectrum disorder. Two prior studies evaluated the impact of monetary reward on vLTM (Calhoun, 1970; Calhoun \& Moss, 1981). In comparisons that lacked healthy control participants, rewards were shown to improve vLTM in institutionalized patients. The more recent of these studies (Calhoun \& Moss, 1981) used a variety of rewards in addition to money (e.g., cigarettes, food), which may have differentially influenced performance and obscured the neuromodulary substrates. More germane was the first of the studies (Calhoun, 1970), which included only monetary reward and examined its differential impact across now obsolete diagnostic classifications (i.e., reactive paranoid, reactive nonparanoid, and process nonparanoid). Interestingly, patients diagnosed with process nonparanoid schizophrenia, who presumably suffered greater negative symptoms, exhibited the most improved word recall with performance-contingent monetary rewards.

More recent work has addressed the impact of reward on executive functions in schizophrenia. With the goal of determining the potential to rehabilitate patient's frontal-executive deficits, investigations into the impact of monetary rewards on the Wisconsin Card Sorting Test (WCST) have generated equivocal or even negative results (Bellack, Mueser, Morrison, Tierney, \& Podell, 1990; Green, Satz, Ganzell, \& Vaclav, 1992; Hellman, Kern, Neilson, \& Green, 1998; Summerfelt et al., 1991; Vollema, Geurtsen, \& van Voorst, 1995; for a review see Kurtz, Moberg, Gur, \& Gur, 2001). Interpretive difficulties arise, however, including the lack of healthy control groups, small samples or subsamples of participants, and the utilization of less sensitive between-sample comparisons. Importantly, the research designs of these studies have often involved multiple WCST administrations. This practice very likely mitigates the typical requirements of the task, because of the increasing likelihood that the sorting principles will be discovered. Indeed, the WCST has been described as a "one-shot" test, suitable only for single administration (Lezak, Howieson, Loring, Hannay, \& Fischer, 2004, p. 588). In summary, although earlier work has addressed the issues of reward and motivation, in the neuropsychology of schizophrenia, the findings are limited.

Consequently, the interactions between reward, symptomatology, and neurocognition in schizophrenia requires further elucidation, particularly in light of the centrality of dopamine dysregulation in the disorder and its importance in both the reward system and neurocognition. Toward these ends, we evaluated the impact of monetary rewards on verbal working memory (vWM) and episodic vLTM in chronic patients exhibiting various symptom dimensions. The selection of these neurocognitive domains was intentional in that both working memory (Conklin, Curtis, Calkins, \& Iacono, 2005; Goldberg et al., 2003; Silver, Feldman, Bilker, \& Gur, 2003; Snitz, MacDonald, \& Carter, 2006) and vLTM (Sponheim, Steele, \& McGuire, 2004; Toulopoulou, Morris, Rabe-Hesketh, \& Murray, 2003; Toulopoulou, Rabe-Hesketh, King, Murray, \& Morris, 2003; Wittorf, Klingberg, \& Wiedemann, 2004) have been proposed as cognitive endophenotypes for schizophrenia on the basis of their severity, heritability, stability (i.e., state independence), and prevalence within the disorder (see Gottesman \& Gould, 2003). Because stability is a criterion for endophenotypes, close examination of the malleability of vWM and vLTM is particularly fitting.

In the current research, gains in neurocognitive performance made in response to reward were indexed against that of healthy control participants. In addition, the contributions of psychiatric symptoms were evaluated. We were particularly interested in whether rewards were less effective performance enhancers in patients exhibiting pronounced negative symptoms (Crow, 1980), specifically, psychomotor poverty (Liddle, 1987). Individuals with these symptoms appear to lack motivation and also to suffer from more prominent vLTM and working memory impairments (Bozikas, Kosmidis, Kioperlidou, \& Karavatos, 2004; Gladsjo et al., 2004; Heydebrand et al., 2004; Liddle, 1987; O'Leary et al., 2000; Pantelis et al., 2004; Rund et al., 2004; Wittorf et al., 2004; Woodward, Ruff, Thornton, Moritz, \& Liddle, 2003). As such, we predicted that patients with marked psychomotor poverty symptoms may be less apt to benefit from reward because of their diminished cortical dopamine availability (Abi-Dargham, 2004; Davis et al., 1991).

Two additional influences were considered in exploratory analyses: the integrity of prefrontal-striatal functioning as indexed by the WCST (Milner, 1963; Stuss et al., 2000) and medication treatment effects. The WCST was chosen because of its relatively limited construct overlap with the measures used in our reward protocols, as well as its association with dopamine neurotransmission and sensitivity to frontal-striatal circuitry (Meyer-Lindenberg et al., 2002; Monchi, Petrides, Petre, Worsley, \& Dagher, 2001). Indices capturing psychopharmacological aspects of the treatment compounds (i.e., anticholinergic load and dopamine blockade) were also examined. Reward-induced changes in memory operations, or the absence of such change, might be wholly or partially treatment induced (Barch, 2005; Juckel, Schlagenhauf, Koslowski, Filonov, et al., 2006).

\section{Method}

\section{Participants}

This research was approved by the ethics review board at Simon Fraser University. All participants were informed of the nature of the study and consented to participate. Fifty tertiary psychiatric inpatients with a diagnosis of schizophrenia $(n=39)$ or schizoaffective $(n=11)$ disorder, as based on the criteria from the Diagnostic and Statistical Manual of Mental Disorders (fourth edition [DSM-IV]; American Psychiatric Association, 1994), and 52 community-dwelling controls were enrolled in the study. The mean duration of patient illness was 14.5 years $(S D=10.9)$. 
Excluding 5 participants on depot medication and 1 patient on amisulpride for which data were unavailable, patients were receiving antipsychotic treatment equivalent to a mean chlorpromazine dosage of 864.6 milligrams $(S D=619.7)$ at the time of testing (Bezchlibnyk-Butler \& Jeffries, 2000). Nonafflicted control participants responded to invitations and postings in the hospital and university community requesting control volunteers for a study examining memory functioning in patients with schizophrenia. With the exception of 1 participant who reported treatment for and recovery from a remote postpartum depression, none of the control participants had been psychiatrically hospitalized. Patients underwent a chart review for medical and psychiatric history and diagnosis. To supplement the patient's chart review and provide health information for controls, we asked all participants to complete a health questionnaire.

All participants were fluent in the English language. Exclusion criteria included mental retardation or pervasive developmental disorders, evidence for active substance abuse at the time of participation, head injury with loss of consciousness for greater than $5 \mathrm{~min}$, or other medical disorders known to significantly influence neuropsychological abilities. All potential participants exhibiting marked intellectual deficits as indicated by a composite IQ of less than 70 on the Kaufmann Brief Intelligence Test (K-BIT; Kaufman \& Kaufman, 1990) at the time of testing were also excluded. Table 1 indicates that the samples were matched on age, parental education (reported by the participants), and gender. ${ }^{1}$

\section{Design}

Participants underwent two experimental protocols as well as neuropsychological testing to quantify select abilities. Persons with schizophrenia also underwent symptom evaluation using the Signs and Symptoms of Psychotic Illness rating scale (SSPI; Liddle, Ngan, Duffield, Kho, \& Warren, 2002) and the WCST. Up to four testing sessions were conducted to accommodate participant test-taking tolerances. The reward protocols were routinely administered in the first portion of the first two sessions.

Neuropsychological battery. All measures were administered according to standard procedures. Intellectual abilities were assessed with the K-BIT. Several established measures of working memory were used, including the Digit Span, Spatial Span, and Letter-Number Sequencing subtests from the Wechsler Memory Scale-Third Edition (WMS-III; Wechsler, 1997b). Information processing speed was evaluated with Symbol Search from the Wechsler Adult Intelligence Test-Third Edition (WAIS-III; Wechsler, 1997a) and the Trail Making Test (Army Individual

Table 1

Demographic Information for all Participants

\begin{tabular}{lcccccccr}
\hline & \multicolumn{3}{c}{$\begin{array}{c}\text { Schizophrenia } \\
\text { spectrum disorder }\end{array}$} & & & \multicolumn{2}{c}{ Control participants } \\
\cline { 2 - 3 } \multicolumn{1}{c}{ Variable } & $n$ & $M$ & $S D$ & & & $M$ & $S D$ \\
\hline Gender: male / female & $32 / 18$ & & & & $29 / 23$ & & \\
Age in years & 50 & 35.9 & 11.86 & & 52 & 32.0 & 11.92 \\
Parental education & 48 & 12.0 & 2.68 & & 52 & 12.6 & 3.64 \\
Participant education & 50 & $11.4^{*}$ & 2.33 & & 52 & $15.0^{*}$ & 1.99 \\
\hline${ }^{*} p<.01$. & & & & & &
\end{tabular}

Test Battery, 1944), Form A. The Trail Making Test, Form B was used to assess speeded executive abilities. As indicated in Table 2, patients performed significantly worse than controls on all the neurocognitive measures.

Experimental reward manipulation. On select neurocognitive tests, participants were informed that they could earn up to $\$ 10$ on the basis of their performance. At the end of the study all participants were "topped up" to the full $\$ 10$ regardless of their actual performances on the rewarded tasks. Each experimental protocol involved administering the same task under a nonrewarded (baseline) and a rewarded condition.

Verbal working memory. The $n$-back task requires fundamental working memory processes, including maintenance, updating, and manipulation of information, and elicits robust activation of frontal and parietal cortices (Owen, McMillan, Laird, \& Bullmore, 2005). The $n$-back task manipulates working memory load by requiring the identification of whether or not the current stimulus had occurred at a fixed number of positions back in the sequence (n-back). Load was manipulated by increasing the number of positions back for which successive identifications were required from no load (0-back) to 4-back. For instance, at 0-back participants indicated whether the current letter is the same ("yes") or different ("no") from a prespecified target letter, at 1-back they successively identified whether the current letter was the same or different from the immediately preceding letter, whereas at 2-back they successively indicated whether the current letter was the same or different from the letter that occurred 2-back in the sequence. Performance on the $n$-back was determined by the total number of correct identifications.

The $n$-back experimental task was administered by computer with Super Lab Pro Version 2.0 (Cedrus, 1999). The betweenletter interstimulus intervals was $50 \mathrm{~ms}$, and letters appeared on the screen for $5,000 \mathrm{~ms}$. The task was administered by alternating rewarded and nonrewarded trial sets across increasing levels of vWM load. In the same session, consecutively recruited participants were counterbalanced to initially receive either the baseline or the rewarded trials and then to receive one of two reward levels. While alternating the rewarded and nonrewarded trial sets at each load level, load was increased from the initial trial set of 0-back to 1-back, 2-back, and 3-back. Control participants were administered an additional 4-back load level (see below for details).

On rewarded trials, a text message appeared prior to the trial informing participants of the breakdown of rewards. This message indicated that for perfect performance a $\$ 1.00$ (high reward) or $\$ 0.50$ (low reward) payout would be earned and that each error resulted in a successive 5-cent decrease in reward earnings. After the rewarded trial sets, a text message was displayed informing the participants of the completion of the "money" trial. Additionally, after each rewarded trial participants were informed of their winnings by the examiner who counted and placed winnings in a clear

\footnotetext{
${ }^{1}$ The actual numbers of control participants in the vWM and vLTM protocols were slightly less than that of the entire sample described, because of experimenter error or discontinuation of a few participants from each of the protocols. Specifically, 48 control participants were enrolled in both the vWM and vLTM protocols, but the constituent participants were not identical.
} 
Table 2

Neuropsychological Findings for all Participants

\begin{tabular}{|c|c|c|c|c|c|c|c|}
\hline \multirow[b]{2}{*}{ Variable } & \multicolumn{3}{|c|}{$\begin{array}{c}\text { Schizophrenia spectrum } \\
\text { disorder }\end{array}$} & \multicolumn{3}{|c|}{ Control participants } & \multirow[b]{2}{*}{$d$} \\
\hline & $n$ & $M$ & $S D$ & $n$ & $M$ & $S D$ & \\
\hline \multicolumn{8}{|l|}{ Intelligence (K-BIT) } \\
\hline Vocabulary score & 50 & 89.3 & 9.50 & 52 & 101.5 & 13.35 & $-1.05^{*}$ \\
\hline Matrices score & 50 & 93.7 & 12.68 & 52 & 106.1 & 9.91 & $-1.09^{*}$ \\
\hline Composite score & 50 & 90.6 & 10.63 & 52 & 104.1 & 10.12 & $-1.30^{*}$ \\
\hline \multicolumn{8}{|l|}{ Working memory ${ }^{\mathrm{a}}$} \\
\hline Digit Span Forward & 47 & 9.2 & 1.91 & 50 & 11.3 & 2.20 & $-1.00^{*}$ \\
\hline Digit Span Backward & 47 & 5.3 & 1.90 & 50 & 8.0 & 2.28 & $-1.31^{*}$ \\
\hline Digit Span total & 47 & 14.5 & 3.24 & 50 & 19.3 & 3.72 & $-1.37^{*}$ \\
\hline Spatial Span & 47 & 13.3 & 3.34 & 50 & 17.6 & 2.31 & $-1.51^{*}$ \\
\hline Letter-Number Sequencing & 48 & 7.8 & 3.00 & 49 & 11.6 & 2.38 & $-1.41^{*}$ \\
\hline \multicolumn{8}{|l|}{ Information processing } \\
\hline Symbol Search ${ }^{\mathrm{b}}$ & 47 & 23.6 & 10.43 & 49 & 41.0 & 7.91 & $-1.89^{*}$ \\
\hline Trail Making Test $\mathrm{A}^{\mathrm{c}}$ & 48 & 41.7 & 15.53 & 51 & 24.0 & 7.46 & $-1.47^{*}$ \\
\hline Trail Making Test $\mathrm{B}^{\mathrm{c}}$ & 47 & 123.8 & 66.50 & 51 & 55.3 & 19.62 & $-1.42^{*}$ \\
\hline
\end{tabular}

Note. $\quad \mathrm{K}-\mathrm{BIT}=$ Kaufman Brief Intelligence Test; for all $d s$, negative values indicate impairment in persons with schizophrenia spectrum disorder.

${ }^{\mathrm{a}}$ Working memory measures are from the Wechsler Memory Scale-Third Edition. ${ }^{\mathrm{b}}$ Symbol Search is from the Wechsler Adult Intelligence Scale-Third Edition. ${ }^{\mathrm{c}}$ Trail Making Tests A and B are from the Army Individual Test Battery.

${ }^{*} p<.01$.

receptacle placed in front of the participants. During nonrewarded trials participants completed the $n$-back task without pre- or posttrial reference to reward.

As detailed below, the 4-back load was administered to control participants to facilitate the matching of schizophrenia and control participants on task difficulty. The 0-back and 1-back data as well as the 2-back data for controls were not analyzed because of ceiling effects (recall accuracy for the aforementioned conditions $>90 \%$ ). Operationally, 2-back percent recall in persons with schizophrenia and 3-back percent recall in controls served as the low vWM load. Relative equivalency between these load levels was indicated by the statistical nonsignificance observed for 2-back overall recall accuracy of patients $(84.6 \%)$ compared with 3-back accuracy of control participants $(84.3 \%)$ across the rewarded and nonrewarded conditions. Likewise, high vWM load was operationalized by the overall accuracy of patients at 3-back $(74.9 \%)$ and controls at 4 -back $(70.6 \%)$ across the rewarded and nonrewarded conditions. These performances did not differ significantly. This matching strategy increases the likelihood that the underlying prefrontal circuitry involving working memory in controls was duly engaged in patients (Van Snellenberg, Torres, \& Thornton, 2006).

Verbal long-term memory. To evaluate the impact of monetary rewards on vLTM, select trials of the California Verbal Learning Test-Second Edition (CVLT-II; Delis, Kramer, Kaplan, \& Ober, 2000) were administered. The CVLT-II is a verbal list-learning test in which a series of 16 items from four categories are repeated over multiple learning and recall trials. After the learning trials, a new list of items is repeated once, and participants are required to recall as many of the new items as they can remember. Then, without repetition of the lists, participants engaged in short- and long-delay free recall and recognition trials in which they are asked to recall the initial list.

Two sessions were conducted, in which the different but equivalent published CVLT-II forms (i.e., Delis et al., 2000) were used to minimize practice effects across consecutive testing sessions (Benedict \& Zgaljardic, 1998; Benedict, 2005), which occurred on different days. In the current paradigm, participants completed the first three learning trials, short- and long-delay free recall, and forced-choice recognition. In one session a nonrewarded baseline performance was established, and in the other session performance under reward was observed. Because of the challenges of scheduling control participant sessions, these participants were evaluated at a significantly longer CVLT-II retest lag $(M=4.7$ days, $S D=3.16)$ compared with schizophrenic participants $(M=3.3$ days, $S D=1.47$ ).

Participants were successively assigned to three counterbalanced VLTM conditions: reward order (baseline to reward vs. reward to baseline), reward level (5 cents vs. 10 cents; low vs. high, respectively), and form order (standard to alternate vs. alternate to standard). With this counterbalancing scheme, the fixed block size was 8 patients. To negate a confound between reward order and symptoms of reality distortion revealed on the SSPI (see below), the final block of 8 patients were assigned to order on the basis of their reality distortion scores. Patients were otherwise randomized for this block.

During baseline and rewarded sessions, participants were tested under standard CVLT-II instructions. Additionally, during the rewarded sessions participants were also told, "for each word you remember correctly, you will receive five (or ten) cents." After each trial in the rewarded session, recall earnings were counted by the examiner and placed into a clear receptacle.

Associated variables. As indicated in the introduction, we were particularly interested in the extent to which psychiatric symptoms predicted reward-related gains in memory performance. Additionally exploratory analyses were undertaken to examine whether these gains were associated with frontal-striatal functioning and pharmacology indices. Finally, we explored the relationships between reward-related memory gains and educational attainment and K-BIT Composite IQ in the total sample as well as 
separately in the subgroups of participants. Separate subgroup analyses were conducted because illness-related factors are apt to influence educational attainment and IQ in the patients (e.g., Aylward, Walker, \& Bettes, 1984; Swanson, Gur, Bilker, Petty, \& Gur, 1998), whereas other mechanisms are responsible for education and IQ variations in healthy persons.

Symptomatology. In persons with schizophrenia, psychopathology was assessed using the SSPI. The SSPI is a 20-item 5 -point criterion-referenced rating scale that is completed after a 25- to 30-min semistructured interview with 15 direct questions about symptoms. Each item is rated from 0 (no pathology) to 4 (severe pathology). The following core items from the SSPI were used to quantify the three-syndrome model of psychotic illness from our previous factor-analytic work: underactivity, flattened affect, and poverty of speech for psychomotor poverty, inappropriate affect and disordered form of thought for disorganization, and delusions and hallucinations for reality distortion (Woodward et al., 2003; Woodward, Thornton, Ruff, Moritz, \& Liddle, 2004). Table 3 illustrates the SSPI per-item ratings for each syndrome and the item scores for the syndrome scales.

Frontal-striatal functioning. Participants with schizophrenia were administered the WCST (Heaton, 1981), a measure that involves frontal-striatal circuitry (e.g., Monchi et al., 2001). The number of perseverative errors was used as the performance indicator. To decrease the influence of several outliers on the WCST, these data underwent logarithmic transformation (log 10). (Note that 7 participants failed to complete the WCST because they discontinued testing.) Additionally, intellectual functioning as indexed by the K-BIT provided an omnibus cognitive comparator not explicitly linked to frontal-striatal functioning. Specifically, the K-BIT Composite IQ was used to assess whether overall intelligence, as opposed to frontal-striatal functioning per se, was associated with reward-related memory gains.

Pharmacology indices. The potential impact of the anticholinergic properties of select study medications and adjunctive compounds was evaluated using two recently established anticholinergic indices developed and validated by Minzenberg, Poole, Benton, \& Vinogradov (2004). The anticholinergic scales consist of (a) a pharmacological index of in vitro muscarinic receptor antagonism and (b) a clinical index of the extent to which compounds

Table 3

Signs and Symptoms of Psychiatric Illness Scale (SSPI) in the Patient Sample

\begin{tabular}{lccc}
\hline & \multicolumn{3}{c}{ Schizophrenia spectrum disorder } \\
\cline { 2 - 4 } SSPI subscale and item & $M$ & $S D$ & Rating range \\
\hline Reality distortion & 1.96 & 1.30 & $0-4.0$ \\
$\quad$ Hallucinations & 1.40 & 1.73 & $0-4.0$ \\
$\quad$ Delusions & 2.52 & 1.39 & $0-4.0$ \\
Disorganization & 0.75 & 0.83 & $0-3.0$ \\
Inappropriate affect & 0.36 & 0.80 & $0-3.0$ \\
$\quad$ Disordered thought form & 1.14 & 1.12 & $0-3.0$ \\
Psychomotor poverty & 1.18 & 0.80 & $0-2.7$ \\
$\quad$ Underactivity & 1.46 & 1.13 & $0-3.0$ \\
Flattened affect & 1.48 & 1.13 & $0-4.0$ \\
Poverty of speech & 0.60 & 0.86 & $0-3.0$ \\
\hline
\end{tabular}

Note. All SSPI scores are provided in per item average units from 0 (no pathology) to 4 (severe pathology). The reported rating range is the observed range in the current sample. induce anticholinergic side effects. For these scales, the anticholinergic load equivalent to $1 \mathrm{mg}$ /day benztropine was calculated for each medication given to each patient (Minzenberg, Poole, Benton, \& Vinogradov, 2004). We supplemented the number of patients that could be included in the pharmacological index by inspecting muscarinic receptor affinities for several additional drugs that were not reported in the Minzenberg et al. (2004) study. Specifically, for one antipsychotic compound ( $n=1$ case) and three antidepressant drugs ( $n=7$ cases), we determined that the anticholinergic load was noncontributory on the basis of affinities obtained from the National Institutes of Health Psychoactive Drug Screening Program (PDSP) Ki database. ${ }^{2}$ Finally, to ensure that estimates were as accurate as possible, persons receiving depot antipsychotic treatments ( $n=5$ cases) were excluded from these analyses. See the Appendix for further details.

To evaluate whether D1 and D2 receptor blockade of the antipsychotic medications influence memory gains, we used a similar strategy to calculate load according to a pharmacological index. The dopamine D1 and D2 receptor affinities for each antipsychotic drug were obtained from the NIH (PDSP) Ki database. The values used were for human cloned receptors, with SCH23390 as the reference ligand for dopamine D1 and raclopride as the reference for dopamine D2. For the study medications, the PDSP-certified Ki value was used where available; otherwise, the mean of the values derived from the published literature was used. The study drug $\mathrm{Ki}$ values were converted to loxapine equivalents and the dopamine D1 and D2 receptor loads were calculated on the basis of total dosage. As with anticholinergic load, 5 persons receiving depot treatments were excluded from these analyses. Additionally, D1 and D2 receptor affinities were unavailable for three compounds ( $n=3$ cases); consequently, these participants' data were excluded from this analysis. See the Appendix for further details.

Data preparation. As described below, five reward-induced memory change (RIMC) indices were calculated to capture the effect of the reward on vWM and vLTM. These indices involved subtracting the baseline (nonrewarded) memory percentage correct from that in the rewarded condition. The percentage correct for a particular measure was each patient's obtained score divided by the total possible score on the measure. The general RIMC formula for RIMC is as follows, where RMS is the rewarded memory score of the participant, TPS $=$ total possible score on the measure, and $\mathrm{BMS}=$ baseline score:

$$
R I M C=[(R M S / T P S) \times 100]-[(B M S / T P S) \times 100]
$$

Thus, an RIMC of $0 \%$ change indicates that the same percentage of the total items remembered in the rewarded condition was remembered at baseline. Positive values indicated improved memory performance under the rewarded condition; negative values indicated poorer performance under reward. The specific reward indices for the vWM and VLTM are further described below.

Two RIMC indices were calculated for vWM, one for low load and a second for high load, by subtracting the percentage of the total number of items recalled in the nonrewarded condition from the percentage of the total number of items recalled in the rewarded condition. These RIMC indices subsequently served as dependent

\footnotetext{
${ }^{2}$ Further information on the Ki database is available at http://pdsp .med.unc.edu.
} 
variables. Additionally, three vLTM indices were calculated to capture RIMC change in learning, immediate recall, and retention. These indices were based on each of the following memory scores: Learning (percent gain over Trials 1 to 3) was measured by calculating the number of items recalled on Trial 1 divided by the total number of possible items, which was subtracted from the items recalled on Trial 3 divided by the total number of possible items, multiplied by 100; Immediate recall (percent recalled for Trials 1 to 3 ) was determined as the total number of items recalled of Trials 1-3 divided by the total number of possible items multiplied by 100 . Finally, Retention (i.e., percentage lost at long-delay free recall) was measured by calculating the short-delay freely recalled items divided by the total number of possible items, which was subtracted from the long-delay freely recalled items divided by the total number of possible items, multiplied by 100 .

In contrast to the highly correlated simple immediate and delayed CVLT-II recall scores, the derived learning, immediate recall, and retention scores were not statistically associated with each other (all $p s>.15$ ), suggesting their relative independence. The products of the RIMC calculations produce results equivalent to that observed when the comparable nonpercentage memory scores are used but have the advantage of being on a metric of $0 \%$ to $\pm 100 \%$. Indeed, whether considering learning and retention performance, each word recall difference or change translated into a $6.25 \%$ difference in score (i.e., 1 word difference from a base of 16 words multiplied by 100); whereas for immediate recall, each word recall difference or change in score translated into a $2.08 \%$ difference in score (i.e., 1 word difference from a base of 48 words multiplied by 100).

For all participants, distributions were examined for extreme values and departures from normality. With one exception, the data observations for all variables were reasonably symmetrical and acceptable for parametric analyses. One retention observation emerged as an extreme value (greater than 4 standard deviations from the mean value). Consequently, an extreme RIMC retention value for this participant was observed. This observation was excluded from the analyses reported below. ${ }^{3}$

\section{Results}

Analyses were conducted (a) to evaluate the differential impact of reward (i.e., RIMC) on vWM and vLTM in patient and control subjects, (b) to predict RIMC from psychiatric symptom ratings, and (c) explore the association between WCST and pharmacological indices on RIMC. All analyses were executed with SPSS 14.

\section{Verbal Working Memory}

Reward-induced memory change (RIMC) was initially analyzed in a $2 \times 2 \times 2 \times 2$ mixed factorial ANOVA, with group (schizophrenia or control), reward level (low vs. high), and reward order (baseline initially vs. reward initially) as the between-subjects variables and vWM load (low vs. high) as a within-subject variable. All variables were fixed, as there was no intention to generalize beyond the defined levels of any of the independent variables. Neither reward order nor its higher order interactions were significant; consequently, this variable was dropped from the analysis. The final model involved a $2 \times 2 \times 2$ analysis of variance (ANOVA), where group, reward level, and load served as the independent variables. ${ }^{4}$
As illustrated in Figure 1, a significant Group $\times$ Load interaction suggests that schizophrenic and control participants exhibited differential RIMC across levels of vWM load, $F(1,94)=4.11$, $p<.05$. Additionally, there was a significant main effect for $\mathrm{vWM}$ load, $F(1,94)=4.67, p<.04$, indicating greater RIMC gains in the high-load condition. Reward level and its higher order interactions were all nonsignificant (all $p \mathrm{~s}>.25$ ); consequently, this variable was eliminated from the subsequent follow-up analyses reported below.

To further evaluate the interaction between group and load, we conducted two repeated measures ANOVAs separately for each group, with load as the independent variable. The intercept was included in the models to test the overall RIMC (i.e., grand mean) against no change in memory. For the schizophrenic group, load did not significantly impact RIMC, $F(1,49)=0.01$, $n s$, and there was no evidence $(d=-.08)$ for overall reward-induced vWM change, $F(1,49)=0.31$, ns.

In contrast, load was a significant moderator of RIMC in controls, $F(1,47)=7.47, p<.01$, indicating that greater gains were made at higher vWM load. Therefore, the intercepts for low and high loads were individually analyzed to determine whether they differed significantly from zero (i.e., no gain). Although at low load, RIMC did not significantly differ from zero $(d=-.19), F(1$, $47)=1.78, n s$, at high load, vWM reward facilitation was induced $(d=.40)$ in control participants, $F(1,47)=7.70, p<.01$.

\section{Verbal Long-Term Memory}

Reward-induced memory change scores for learning, immediate recall, and retention was analyzed separately in three $2 \times 2 \times 2$ factorial ANOVA with group (schizophrenia or control), reward level (low vs. high), and reward order (first vs. second session) as the between-subjects variables. The intercept was included in the model to evaluate the RIMC against zero, indicative of no change in memory. All variables were fixed. Neither reward order nor its higher order interactions were significant for any of the dependent variables; consequently, reward order was dropped from the analyses. The final models were three $2 \times 2$ ANOVAs, where group and reward level served as the independent variables. ${ }^{5}$

According to the intercept values, there were no significant RIMC increases in learning $(d=.09), F(1,94)=0.82, n s$, or immediate recall $(d=-.02), F(1,94)=0.02$, $n s$. Furthermore, there was an absence of differential effects for group, all $F \mathrm{~s}(1$, $94)<0.4, n s$, and reward level, all $F_{\mathrm{s}}(1,94)<.10$, $n s$, for both of these independent variables. In contrast, Figure 2 indicates that

\footnotetext{
${ }^{3}$ Nonetheless, the data were evaluated after adjusting this extreme vLTM retention value to make it contiguous with the next closest value while maintaining its distal-most ranking in the distribution (Tabachnick \& Fidell, 2007). Unless otherwise indicated, the results reported below with the extreme value excluded were consistent with the results obtained from this adjustment to the data.

${ }^{4}$ ANCOVAs with educational attainment and intellectual functioning as covariates and group and load as the independent variables were also conducted. All covariates were clearly nonsignificant $(p s>.50)$ and were therefore not included in any of the models.

${ }^{5}$ ANCOVAs with educational attainment, intellectual functioning, and retest lag as covariates and group and load as the independent variables were also conducted. All covariates were clearly nonsignificant (all ps > .14) and were therefore not included in any of the models.
} 
vWM Reward Induced Memory Change
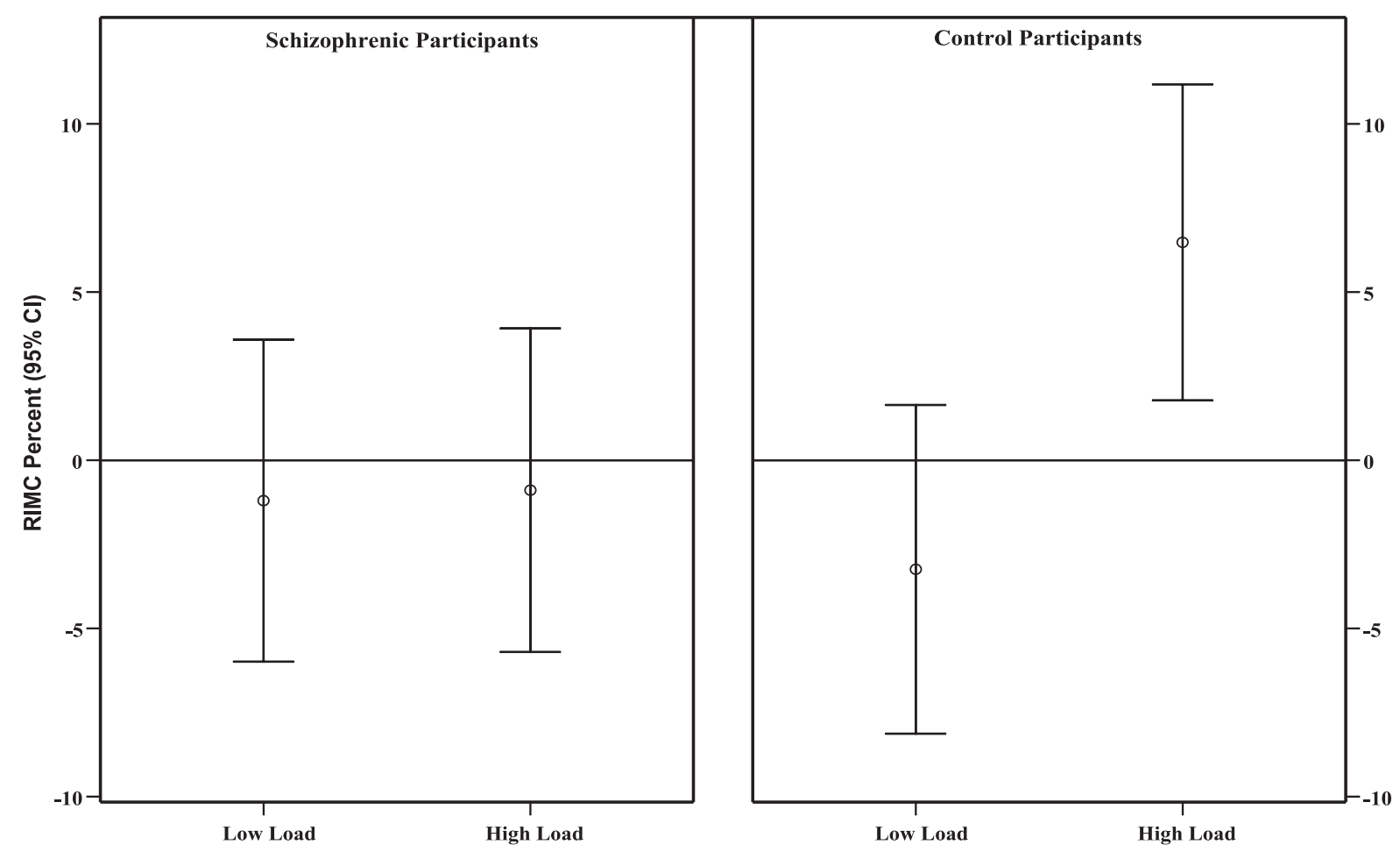

Figure 1. Reward-induced memory change (RIMC) as a function of verbal working memory (vWM) load in schizophrenic and control participants.

a significant facilitation was induced by reward for vLTM retention $(d=.35)$ in the entire participant sample, $F(1,93)=11.6$, $p=.001$. This effect was indistinguishable in patients versus controls, group $F(1,93)=0.41, n s$. Furthermore, there was no difference in RIMC scores at the two reward levels, $F(1$, $93)=1.00, n s$, nor was there a Group $\times$ Reward Level interaction, $F(1,93)=0.05, n s$.

\section{Symptom Analyses}

To evaluate whether differential RIMC was associated with the symptoms of schizophrenia, we conducted regression analyses using the SSPI symptom dimensions scores as predictors. Specifically, we conducted four analyses to predict RIMC for overall vWM (i.e., mean vWM from the high and low loads) and for vLTM learning, immediate recall, and retention. All three SSPI symptoms predictors (i.e., reality distortion, psychomotor poverty, and disorganization) were entered on a single step into the preliminary models; subsequently, only the significant predictors were retained in the final models, which are reported in Table 4. Note that none of the SSPI predictors were significant for RIMC in learning, so no information from the learning model is reported.

As can be seen in Table 4, the extent of reality distortion symptoms was differentially associated with the RIMCs observed for vWM and immediate VLTM recall. Interestingly, increased reality distortion was associated with gains in overall vWM but losses in immediate vLTM. In the absence of reality distortion symptoms (i.e., no pathology, SSPI rating of zero), patients expe- rienced a significant reward-related $\mathrm{vWM}$ decline of $7.3 \%$ from their nonrewarded baseline (see Intercept Analysis 1, Table 4), but for each point increase in reality distortion, a $3.2 \%$ gain in $\mathrm{vWM}$ was observed. This translated into a modest $5.5 \%$ gain in vWM at the maximum and most severe level of reality distortion (SSPI rating of 4).

In contrast, in the absence of reality distortion symptoms, the same patients exhibited a significant reward-related gain in immediate recall from vLTM of 5.5\% (see Intercept Analysis 2, Table 4). For each point increase in reality distortion, a loss of $2.6 \%$ in immediate recall from vLTM was indicated by the analysis. Thus, on average, a $4.9 \%$ loss in immediate vLTM occurred at the most severe level of reality distortion.

Additionally, higher psychomotor poverty and disorganization symptoms were associated with reduced reward-elicited memory retention gain. When both psychomotor poverty and disorganization were absent, the model predicted a $15.4 \%$ gain in vLTM retention on average (see Intercept Analysis 3, Table 4). Furthermore, each point increase in disorganization translated into a $4.8 \%$ loss in retention, and each point increase in psychomotor poverty translated into a $7.1 \%$ retention loss.

\section{Exploratory Analyses}

For patient data, correlations were conducted between RIMC and frontal-striatal function, as indexed by perseverative errors on the WCST, as well as select pharmacological aspects of the treatments. Additionally, for the total sample of participants and the participant subgroups, correlations were conducted between 
vLTM Reward Induced Memory Change
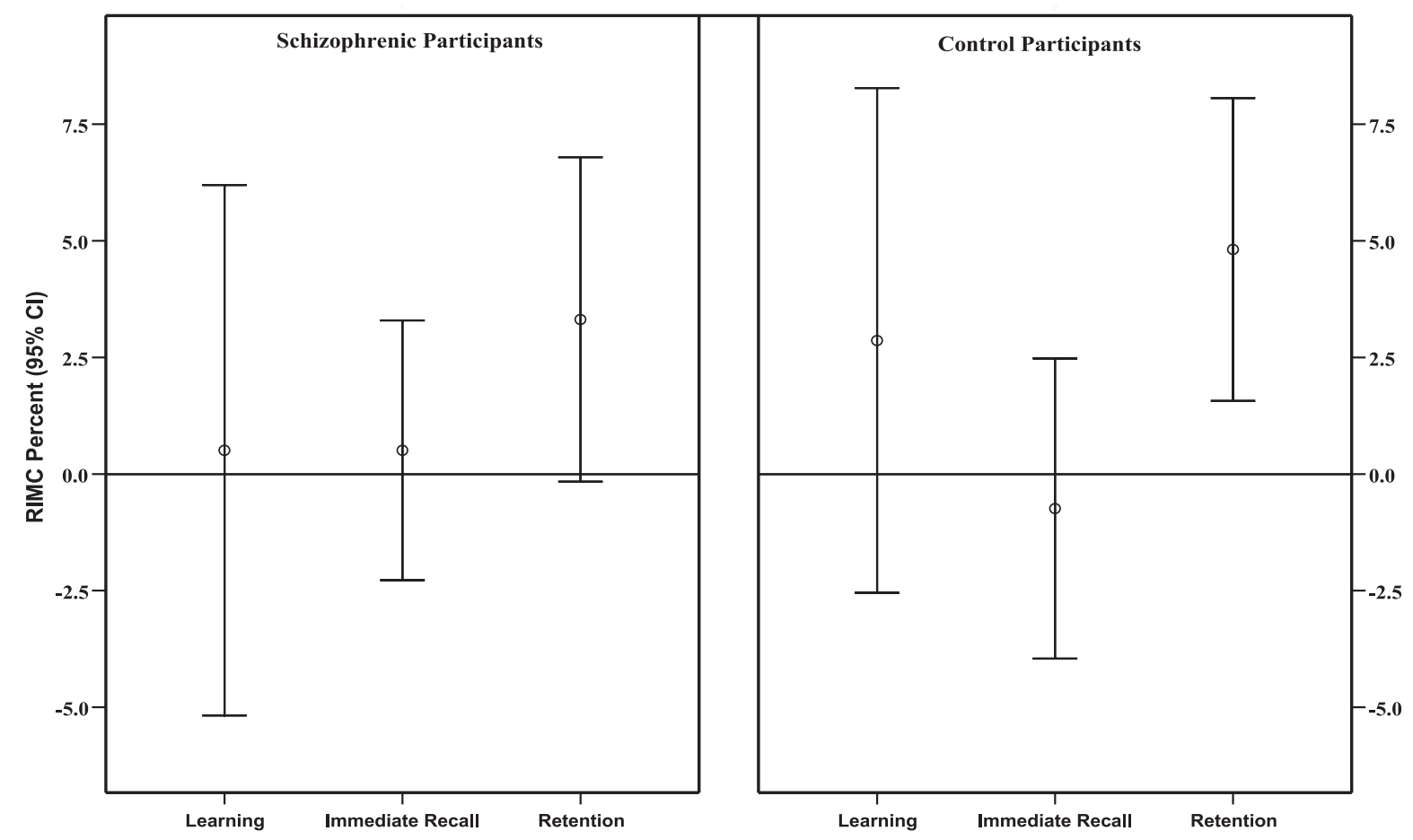

Figure 2. Reward-induced memory change (RIMC) across three aspects of verbal long-term memory (vLTM) in schizophrenic and control participants.

RIMC and overall intellectual functioning (Composite K-BIT) and educational attainment. These results are presented in Tables 5 and 6, and select RIMC patient findings are further illustrated in Figure 3.

Figure 3A illustrates that increasing frontal-striatal dysfunction, as indexed by WCST perseverative errors, was associated with

Table 4

Final Regression Models Predicting Reward-Induced Memory Change Scores on the Basis of the Signs and Symptoms of Psychiatric Illness (SSPI) Scale

\begin{tabular}{clllllll}
\hline SSPI scale & $B$ & $S E$ & $\beta$ & $s r^{2}$ & $t$ & $p$ \\
\hline
\end{tabular}

Analysis 1: Verbal working memory total

\begin{tabular}{lrrrrrr} 
Reality distortion & 3.20 & 1.39 & .32 & .10 & $2.30^{\mathrm{b}}$ & .026 \\
Intercept & -7.31 & 3.26 & & & $-2.25^{\mathrm{b}}$ & .029 \\
\hline
\end{tabular}

Analysis 2: Verbal long-term memory immediate recall

$\begin{array}{lrrrrrr}\text { Reality distortion } & -2.59 & 1.00 & -.35 & .12 & -2.59^{\mathrm{b}} & .013 \\ \text { Intercept } & 5.49 & 2.34 & & & 2.34^{\mathrm{b}} & .023\end{array}$

Analysis 3: Verbal long-term memory retention ${ }^{\mathrm{a}}$

\begin{tabular}{lrrrrrr} 
Psychomotor poverty & -7.07 & 2.44 & -.47 & .15 & $-2.90^{\mathrm{c}}$ & .006 \\
Disorganization & -4.76 & 2.36 & -.33 & .07 & $-2.02^{\mathrm{c}}$ & .050 \\
Intercept & 15.37 & 4.48 & & & $3.43^{\mathrm{c}}$ & .001 \\
\hline
\end{tabular}

${ }^{a}$ When the extreme value was adjusted (rather than removed), the significance of disorganization was lost and psychomotor poverty became marginally significant; nonetheless, the intercepts remained significant. ${ }^{\mathrm{b}} \mathrm{df}=48{ }^{\mathrm{c}} \mathrm{df}=46$. decreases in reward-related vLTM retention gains. In contrast, overall intellectual functioning was not associated with RIMC in any evaluated domain (see Table 5).

In terms of medication parameters, Figure $3 \mathrm{~B}$ indicates that RIMC gains in VLTM retention were associated with lower relative anticholinergic load on the pharmacological index. Marginal trends in the same direction were noted on the clinical index of anticholinergic load. Gains in vLTM retention also varied as a function of D1 blockade; lesser blockade was associated with greater memory gains (see Table 5). However, the dopamine and anticholinergic indices were all significantly intercorrelated (all $p s<.001)$, suggesting considerable statistical overlap in these pharmacology measures.

To address the potential that D1 blockade and/or anticholinergic load induced psychomotor symptoms, which in turn produced a surrogate relationship between psychomotor poverty and vLTM retention (see Analyses 3, Table 4), we conducted a partial correlation analysis between psychomotor poverty and vLTM retention, controlling for both D1 blockade and anticholinergic load. Despite this statistical control, the association between attenuated vLTM gains and increasing psychomotor poverty symptoms remained $(r=-.35, p<.05)$, indicating that these medication parameters did not underlie this relationship.

Finally, we evaluated the association between RIMC and overall intellectual functioning and educational attainment in the total sample as well as separately for the patient and control groups. Table 6 illustrates that the only statistically significant associations observed for the total sample were for vWM gains. Specifically, higher educational attainment and stronger intellectual abilities were both associated with greater gain in $\mathrm{vWM}$ 
Table 5

Pearson Correlation Coefficients Between RIMC and Moderating Factors in Patients

\begin{tabular}{|c|c|c|c|c|c|c|c|c|}
\hline \multirow[b]{2}{*}{ Variable } & \multicolumn{2}{|c|}{$\begin{array}{l}\text { vWM } \\
\text { (overall) }\end{array}$} & \multicolumn{2}{|c|}{ Learning } & \multicolumn{2}{|c|}{$\begin{array}{l}\text { Immediate } \\
\text { recall }\end{array}$} & \multicolumn{2}{|c|}{ Retention } \\
\hline & $r$ & $n$ & $r$ & $n$ & $r$ & $n$ & $r$ & $n$ \\
\hline D1 blockade & -.04 & 42 & .08 & 42 & -.18 & 42 & $-.32^{*}$ & 41 \\
\hline D2 blockade & .08 & 42 & .04 & 42 & -.07 & 42 & -.16 & 41 \\
\hline $\begin{array}{l}\text { Anticholinergic } \\
\text { p-Load }\end{array}$ & -.02 & 43 & .08 & 43 & -.25 & 43 & $-.37^{*}$ & 42 \\
\hline $\begin{array}{l}\text { Anticholinergic } \\
\text { c-Load }\end{array}$ & -.05 & 42 & .05 & 42 & -.16 & 42 & $-.31 \dagger$ & 41 \\
\hline $\begin{array}{l}\text { WCST perseverative } \\
\text { errors }\end{array}$ & -.10 & 43 & .03 & 43 & -.20 & 43 & $-.38^{*}$ & 42 \\
\hline $\begin{array}{l}\text { K-BIT Composite } \\
\text { IQ }\end{array}$ & .06 & 50 & .14 & 50 & .03 & 50 & .13 & 49 \\
\hline
\end{tabular}

Note. $\quad \mathrm{vWM}=$ verbal working memory; $\mathrm{p}-$ load $=$ pharmacological load; c-load = clinical load; D = dopamine; WCST $=$ Wisconsin Card-Sorting Test; K-BIT = Kaufman Brief Intelligence Test. The D1 blockade correlation with verbal long-term memory retention was rendered marginally significant when the extreme value was adjusted.

${ }^{\dagger} p<.10 .{ }^{*} p<.05$ (two-tailed).

in the high-load condition for the entire sample. Statistically significant effects for these variables were not observed for patients or for healthy controls when these groups were analyzed separately in the high-load condition. In terms of the subgroups, for persons with schizophrenia, lesser educational

Table 6

Pearson Correlation Coefficients Between RIMC and IQ and Education

\begin{tabular}{|c|c|c|c|c|c|c|}
\hline \multirow[b]{2}{*}{ Measure } & \multicolumn{2}{|c|}{$\begin{array}{l}\text { Total sample } \\
\qquad(n=98)^{\mathrm{a}}\end{array}$} & \multicolumn{2}{|c|}{$\begin{array}{l}\text { Schizophrenia } \\
\quad(n=50)^{\mathrm{b}}\end{array}$} & \multicolumn{2}{|c|}{$\begin{array}{l}\text { Controls } \\
(n=48)\end{array}$} \\
\hline & $\begin{array}{l}\text { Low } \\
\text { load }\end{array}$ & $\begin{array}{l}\text { High } \\
\text { load }\end{array}$ & $\begin{array}{l}\text { Low } \\
\text { load }\end{array}$ & $\begin{array}{l}\text { High } \\
\text { load }\end{array}$ & $\begin{array}{l}\text { Low } \\
\text { load }\end{array}$ & $\begin{array}{l}\text { High } \\
\text { load }\end{array}$ \\
\hline \multicolumn{7}{|c|}{ Verbal working memory } \\
\hline $\begin{array}{l}\text { K-BIT } \\
\text { Education (years) }\end{array}$ & $\begin{array}{l}-.05 \\
-.20\end{array}$ & $\begin{array}{l}.22^{*} \\
.21^{*}\end{array}$ & $\begin{array}{l}-.05 \\
-.40^{* *}\end{array}$ & $\begin{array}{l}.14 \\
.04\end{array}$ & $\begin{array}{l}.01 \\
.04\end{array}$ & $\begin{array}{l}.10 \\
.15\end{array}$ \\
\hline
\end{tabular}

Verbal long-term memory-learning

\begin{tabular}{llll} 
K-BIT & .10 & .14 & .00 \\
Education (years) & .10 & .06 & .07 \\
\hline
\end{tabular}

Verbal long-term memory_immediate recall

\begin{tabular}{lrrr} 
K-BIT & -.02 & .03 & .02 \\
Education (years) & -.06 & -.09 & .02 \\
\hline
\end{tabular}

Verbal long-term memory-retention

\begin{tabular}{lrrr} 
K-BIT & .08 & .13 & -.03 \\
Education (years) & -.06 & .01 & $-.30^{*}$ \\
\hline
\end{tabular}

Note. $\quad \mathrm{K}-\mathrm{BIT}=$ Kaufman Brief Intelligence Test-Composite IQ.

${ }^{a}$ Except for verbal long-term memory retention, where total sample $n=97 .{ }^{\mathrm{b}}$ Except for verbal long-term memory retention, where total sample $n=49$.

${ }^{*} p<.05 .{ }^{* *} p<.01$ (two-tailed).
A.

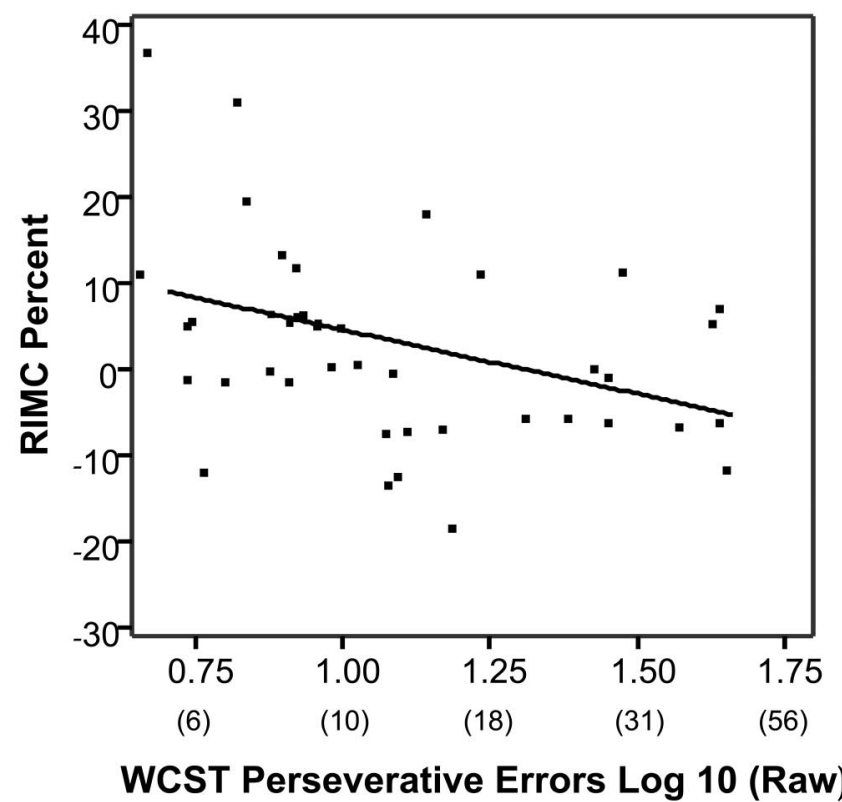

B.

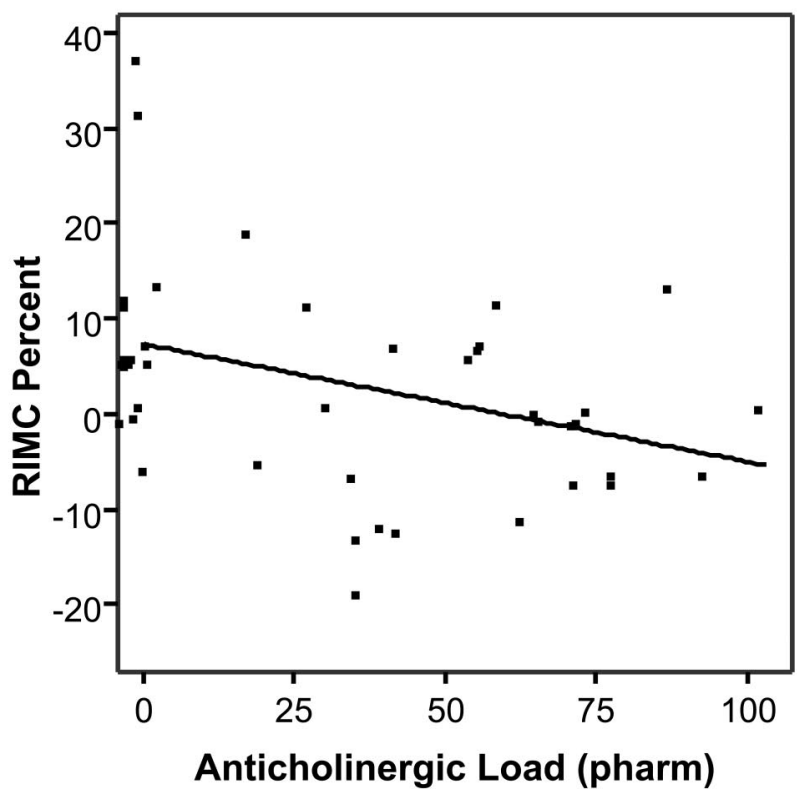

Figure 3. Significant associates of verbal long-term memory (vLTM) retention gains: (A) Wisconsin Card Sorting Test (WCST) and (B) pharmacological index (pharm) of anticholinergic load. To enhance the display of the data points, the data has been "jittered" by adding $4 \%$ random noise to all scale axis dimensions.

attainment was associated with greater gains in vWM in the low-load condition. Additionally, for control participants, lesser educational attainment was associated with enhanced vLTM retention gains. 


\section{Discussion}

The interface between select aspects of neurocognition and monetary reward was evaluated in a sample of healthy individuals and in persons with schizophrenia spectrum disorder. The findings indicated that contingent monetary rewards delivered during vWM and vLTM effectively impacted aspects of memory performance. As detailed below, the influence of reward was limited. Furthermore, reward effects were dependent on the specific neurocognitive operation examined, the mental health status of the participants, and their symptom manifestations and pharmacological treatments.

\section{Verbal Working Memory}

In the first experiment, persons with schizophrenia failed to exhibit vWM improvement over their nonrewarded baseline. In contrast, at a higher level of vWM difficulty, healthy individuals increased their recall accuracy by approximately $6.5 \%$ when they received performance-contingent rewards. Apparently, the impact of reward that resulted in vWM gains in healthy individuals was absent in patients.

Despite the overall lack of reward-related benefit for patients, reward-related $\mathrm{vWM}$ gains were associated with the extent of reality distortion symptoms. Indeed, the absence of reality distortion was associated with reward-related vWM declines from baseline. Importantly, at baseline, patients with low reality distortion (below the median SSPI score) exhibited higher vWM accuracy relative to the accuracy of patients with pronounced reality distortion scores (overall vWM accuracy $84.2 \%$ versus $76.3 \%$, respectively). In the rewarded condition this difference between patients with low and high reality distortion was eliminated $(78.9 \%$ vs. $79.5 \%$, respectively). Thus, to a large degree, the positive association between greater $\mathrm{vWM}$ gains and increased reality distortion symptoms reflected a decline in vWM under reward for patients with higher baseline accuracy and minimal reality distortion symptoms. Patients with low reality distortion symptoms did not exhibit such a decline. This pattern suggests that optimal vWM efficiency was disrupted by reward in the patients lacking reality distortion symptoms. Such an interpretation is analogous to observations indicating that some dopamine agonists may impair working memory in persons with high baseline capacity but have beneficial effects in persons with lower baseline capacity (e.g., Kimberg, D’Esposito, \& Farah, 1997; Mattay et al., 2000). Indeed, the general relationship between dopamine and working memory is nonlinear, with both hyper- and hypodopaminergic activity linked to working memory deficits (see Mehta \& Rimel, 2006; Robbins, 2005; Williams \& Castner, 2006).

The current study also suggested that psychomotor poverty symptoms in the patients were not associated with vWM gains. Negative symptoms, including psychomotor poverty, are purported to involve decreased dopaminergic activity in the cortex (e.g., Abi-Dargham, 2004; Abi-Dargham \& Moore, 2003). These symptoms have been directly associated with working memory deficits arising from the prefrontal cortex (Goldman-Rakic and Selemon, 1997). If a mutual attenuation of dopaminergic neurotransmission impacts both vWM gains and the psychomotor poverty observed in the patients, the threshold at which dopamine deficiencies impede reward-related vWM gains must diverge from that necessary to induce psychomotor poverty symptoms. Otherwise, an association would be expected between increased psy- chomotor poverty and decreasing RIMC gains in vWM. Alternatively, it may be that the dopaminergic dysregulation influencing vWM occurs in different neural regions than that involving psychomotor poverty or that the mechanisms for these phenomena are entirely separate. Whereas the frontal cortex plays a central role in symptoms of psychomotor poverty, these symptoms are associated with subcortical and cerebral anomalies as well (e.g., Galeno, Molina, Guirao, \& Isoardi, 2004; Potkin et al., 2002). For instance, at rest, hyperactivation of subcortical nuclei has been associated with more severe negative symptoms (Galeno et al., 2004). Additionally, negative symptoms are also associated with subcortical abnormalities during reward anticipation. Specifically, when participants with schizophrenia anticipate reward, hypoactivation of the ventral striatum is associated with the severity of negative symptoms (Juckel, Schlagenhauf, Koslowski, Filonov, et al., 2006; Juckel, Schlagenhauf, Koslowski, Wüstenberg, et al., 2006). Thus, symptoms of psychomotor poverty and reward processing might reflect subcortical involvement, whereas the cognitive operations of working memory itself may be more cortically based. Functional neuroimaging protocols designed to directly address this issue will likely be necessary to provide clarification.

The lack of gain in patients' vWM may be either primarily related to the disorder or secondarily related to its treatment. Indeed, antagonism of striatal dopamine may augment negative symptoms of schizophrenia (Heinz et al., 1998). Furthermore, cholinergic blockade is associated with attenuation of attention and memory functioning in schizophrenia (Minzenberg et al., 2004; Thornton, Van Snellenberg, Sepehry, \& Honer, 2006). Although, it is conceivable that antipsychotic treatment dampened vWM reward effects while decreasing psychotic symptoms, the current results failed to detect a relationship between RIMC and the particular psychopharmacology indices used in our study. This suggests that the absence of vWM reward-induced gains at high load is an anomaly that might be a primary form of dysfunction in schizophrenia and not medication induced. Nonetheless, it is also important to note that the methods to index pharmacology parameters were limited. Future researchers should consider experiments in unmedicated patients or use a more controlled approach to the treatment compounds.

\section{Verbal Long-Term Memory}

In contrast to the vWM findings, monetary rewards seemingly had similar impact on vLTM for patients and controls. Specifically, in the entire sample 20-min delayed word retention was increased by approximately $4 \%$ in the rewarded condition but learning and immediate vLTM were not affected significantly. The findings of only a reward-related effect for vLTM retention might reflect short-term memory contamination (i.e., recency recall) in the learning and immediate recall conditions. That is, compared with LTM retention, these two conditions are apt to engage both short-term, recency memory as well as LTM processes. In contrast, the period of distraction and delay entailed in retention chiefly involves LTM processes (see Butters, Delis, \& Lucas, 1995). Prior findings of LTM reward facilitation have utilized only paradigms with significant delays (Adcock et al., 2006; Wittmann et al., 2005) or extensive word lists that are considerably taxing of LTM (Calhoun, 1970).

Whereas the vLTM findings indicated that persons with schizophrenia and controls experience relatively equivalent responses to 
reward, several factors influenced patient gains, suggesting that it is hasty to conclude normalcy in VLTM reward response for all persons with schizophrenia. Specifically, reward-induced improvements in retention were larger in patients with lesser psychomotor poverty, when anticholinergic load and D1 blockade were low, and in patients who exhibited relatively strong frontal-striatal functioning.

Furthermore, the association between psychomotor poverty severity and attenuated reward-associated retention gains was not contingent on dopaminergic or anticholinergic medication effects at least within the limits of the psychopharmacological indices used. Apparently, deficits in vLTM retention are central to the disorder's core negative symptomatology. Relative to patients who lack negativity, individuals with prominent psychomotor poverty may suffer accompanying vLTM retention deficits that are particularly intractable. It is important to recognize that compared with positive aspects of schizophrenia, the negative symptoms are also least responsive to treatment (e.g., Erhart, Marder, \& Carpenter, 2006).

A primary goal of the current study was to elucidate the overall malleability of vWM and vLTM deficits in persons with schizophrenia. Both working memory and vLTM have been proposed as endophenotypes for schizophrenia. The centrality of these impairments to schizophrenia is bolstered by their strong associations to both premorbid functioning (Rund et al., 2004) and functional outcomes (Addington \& Addington, 2000; Green, Kern, Braff, \& Mintz, 2000; Green, 1996; Green, Kern, \& Heaton, 2004; Moritz et al., 2000; Revheim et al., 2006). Overall, the vWM and vLTM neurocognitive abilities under evaluation proved to be reasonably stable. Despite the application of monetary rewards, as a group, participants with chronic schizophrenia exhibited no significant gain in vWM and only a limited gain in vLTM. Indeed, although there was a reward-induced improvement in vLTM retention for the overall participant sample, this effect was relatively small in persons with schizophrenia $(d=.27)$ and only marginally significant, $t(48)=1.92, p=.06$. Consequently, to the extent that the current findings generalize to other patient samples, vWM and vLTM impairments may serve as an endophenotype that provides a link between the genetics and neurophysiology of schizophrenia spectrum disorder and the more complex full phenotypic expressions (see Gottesman \& Gould, 2003).

\section{Limitations}

Five limitations should be considered in evaluating the findings. First, the rewards used in this study cannot be assumed to be experienced as equivalent in patients and healthy individuals. For example, presuming that small monetary rewards have higher saliency for patients, attenuated or equivalent RIMC gains for patients compared with healthy participants may actually imply a deficit in reward response in schizophrenia. On the other hand, it is possible that healthy individuals are better conditioned to respond to symbolic rewards such as money.

Second, our results are constrained by the chronic nature of the sample of schizophrenia patients we enrolled. These patients were institutionalized and under treatment. They may not be representative of the population suffering from this disorder in general. Indeed, it will be important to replicate these findings in a less ill sample and perhaps in unmedicated patients.
Third, divergent rewards and alternative neurocognitive tasks may lead to different conclusions. In this vein, it might be of considerable interest to determine whether certain cognitive operations are more amenable to rewards, particularly functions that are not considered to be cognitive endophenotypes. Such operations may also be more responsive to neurorehabilitation.

Fourth, some potentially important statistically significant differences were observed in patient and control group comparisons. Namely, longer CVLT-II retest lag, greater educational attainment, and higher levels of intellectual functioning were observed in the control group. These differences will be considered individually as they pertain to overall contributions to RIMC and their potential for contributing to the difference observed between the groups in their response to reward.

In the analyses of VLTM, the retest lag of the CVLT-II administrations was approximately 1.4 days longer for control participants than for patients. Nonetheless, this lag was not associated with RIMC (all $p \mathrm{~s}>.10$ ), and coefficients were relatively small, that is, vLTM learning $(r=.17)$, immediate recall $(r=.01)$, and retention $(r=.03)$. Furthermore, retest lag did not emerge as a statistically significant covariate in the ANCOVA models (see Footnote 5).

The issue of educational and IQ differences between the patient and control groups is considerably more complex. Following the suggestions of Resnick (1992), the participant groups were not matched on education (or intellectual abilities); rather, matching was accomplished on the basis of parental education. Importantly, educational attainment and Composite IQ were not significant covariates in the statistical analyses of vWM and vLTM (see Footnotes 4 and 5). Nonetheless, inspection of the zero-order correlations indicated that higher IQ scores and increased years of formal education were associated with greater vWM gains in the high-load condition for the total sample of participants. Statistically significant associations between these variables did not emerge when the schizophrenic and control participants were evaluated in separate correlation analyses. Because RIMC in the high vWM load condition differed between the groups, further consideration of whether group differences in educational and intellectual functioning accounted for differential vWM gain is germane.

It is generally accepted that truncated education and intellectual decline are integral features of schizophrenia itself (see Resnick, 1992; Swanson et al., 1998; Wilk et al., 2005). Rather than being more narrowly associated with scholastic abilities or intelligence, lesser educational attainment is associated with early disease onset and greater symptom severity for persons suffering from schizophrenia. Indeed, lesser education is correlated with more severe positive and negative schizophrenia symptoms (e.g., Swanson et al., 1998). It has also been contended that matching on education may result in mismatching on other relevant variables and that statistical control procedures obscure findings because education is confounded with several illness-related factors (see Buckley, 1992; Resnick, 1992). The same issues likely hold true for intelligence, because in schizophrenia intellectual impairments are associated with more severe illness manifestations and poorer prognosis (e.g., Aylward et al., 1984). Lower intellectual functioning in persons with schizophrenia is a manifestation of the disease, as opposed to primarily reflecting the innate intellectual abilities of these individuals. 
Statistically, it is also unlikely that these variables are the primary mechanisms responsible for the observed group differences in vWM gains. First, when educational attainment and intellectual functioning were included as covariates in the ANCOVA models (see Footnotes 4 and 5), they did not contribute to the models significantly. Second, although significant zero-order correlations were noted between educational attainment, composite intelligence scores, and vWM gains (high load) for the total sample, these associations were absent when patient and control subgroups were evaluated separately. In conjunction with the statistically significant attenuation in the educational and intellectual function of schizophrenia participants, the absence of subgroup correlations is consistent with the contention that educational attainment and intelligence are proxy variables for the presence or absence of the disorder.

A final limitation is that at the neuromodulary level the interactions between reward, cognition, and dopamine are very complex. Neurobiological probes that directly assess the nature of these relationships were not used in the current research. Although the testing paradigms were apt to differentially elicit reward circuitry during the $\mathrm{vWM}$ and vLTM tasks, the regional substrates for our observations await future investigations.

In conclusion, determining how much significance to ascribe to the well-established neurocognitive deficits in persons with schizophrenia who often appear to lack the motivation to fully engage in the task is challenging for clinician and researcher alike. The malleability of the deficits has important diagnostic and rehabilitation implications. The current research suggests that contingent monetary rewards generate only limited improvement in aspects of both vWM and vLTM functioning in healthy persons; in those with schizophrenia, gains appear to be even more limited. Thus, the deficits that persons with chronic schizophrenia experience in vWM and vLTM appear to be relatively invariant, supporting the contention that these functions may represent reasonable neurocognitive endophenotypes for the disorder. The stability of deficits observed suggests that the underlying neurocognitive functions are recalcitrant to simple monetary rewards. For rehabilitation purposes, the extent to which other environmental manipulations can restore these functions remains an important research question, as is the generalizability of the current findings to other patient samples.

\section{References}

Abi-Dargham, A. (2004). Do we still believe in the dopamine hypothesis? New data brings new evidence. The International Journal of Neuropsychopharmacology, 7(Suppl 1), S1-S5.

Abi-Dargham, A., Mawlawi, O., Lombardo, I., Gil, R., Martinez, D., Huang, Y., et al. (2002). Prefrontal dopamine D1 receptors and working memory in schizophrenia. The Journal of Neuroscience: The Official Journal of the Society for Neuroscience, 22, 3708-3719.

Abi-Dargham, A., \& Moore, H. (2003). Prefrontal DA transmission at D1 receptors and the pathology of schizophrenia. The Neuroscientist: A Review Journal Bringing Neurobiology, Neurology and Psychiatry, 9, 404-416.

Adcock, R. A., Thangavel, A., Whitfield-Gabrieli, S., Knutson, B., \& Gabrieli, J. D. (2006). Reward-motivated learning: Mesolimbic activation precedes memory formation. Neuron, 50, 507-517.

Addington, J., \& Addington, D. (2000). Neurocognitive and social functioning in schizophrenia: A 2.5 year follow-up study. Schizophrenia Research, 44, 47-56.
American Psychiatric Association. (1994). Diagnostic and statistical manual of mental disorders (4th ed.). Washington, DC: Author.

Army Individual Test Battery. (1944). Manual of directions and scoring. Washington, DC: War Department, Adjutant General's Office.

Aylward, E., Walker, E., \& Bettes, B. (1984). Intelligence in schizophrenia: Meta-analysis of the research. Schizophrenia Bulletin, 10, 430-459.

Backman, L., Ginovart, N., Dixon, R. A., Wahlin, T. B., Wahlin, A., Halldin, C., et al. (2000). Age-related cognitive deficits mediated by changes in the striatal dopamine system. The American Journal of Psychiatry, 157, 635-637.

Barch, D. M. (2005). The relationships among cognition, motivation, and emotion in schizophrenia: How much and how little we know. Schizophrenia Bulletin, 31, 875-881.

Bellack, A. S., Mueser, K. T., Morrison, R. L., Tierney, A., \& Podell, K. (1990). Remediation of cognitive deficits in schizophrenia. The American Journal of Psychiatry, 147, 1650-1655.

Benedict, R. H. B. (2005). Effects of using same- versus alternate-form memory tests during short-interval repeated assessments in multiple sclerosis. Journal of the International Neuropsychological Society, 11, 727-736.

Benedict, R. H. B., \& Zgaljardic, D. J. (1998). Practice effects during repeated administrations of memory tests with and without alternate forms. Journal of Clinical and Experimental Neuropsychology, 20, $339-352$.

Bezchlibnyk-Butler, K. Z., \& Jeffries, J. J. (Eds.). (2000). Clinical handbook of psychotropic drugs. Seattle, WA: Hogrefe \& Huber.

Bozikas, V. P., Kosmidis, M. H., Kioperlidou, K., \& Karavatos, A. (2004). Relationship between psychopathology and cognitive functioning in schizophrenia. Comprehensive Psychiatry, 45, 392-400.

Buckley, P. (1992). Schizophrenia research-The problem of controls. Biological Psychiatry, 32, 215-217.

Butters, N., Delis, D. C., \& Lucas, J. A. (1995). Clinical assessment of memory disorders in amnesia and dementia. Annual Review of Psychology, 46, 493-523.

Calhoun, J. F. (1970). Effects of performance payoff and cues on recall by hospitalized schizophrenics. Journal of Abnormal Psychology, 76, 485491.

Calhoun, J. F., \& Moss, R. A. (1981). The role of level of functioning in the modification of psychological deficit in schizophrenics. Journal of Psychiatric Research, 16, 205-211.

Carter, C. S. (2005). Applying new approaches from cognitive neuroscience to enhance drug development for the treatment of impaired cognition in schizophrenia. Schizophrenia Bulletin, 31, 810-815.

Cedrus. (1999). SuperLab Experimental Lab Software (Version 2.01). San Pedro, CA: Author.

Conklin, H. M., Curtis, C. E., Calkins, M. E., \& Iacono, W. G. (2005). Working memory functioning in schizophrenia patients and their firstdegree relatives: Cognitive functioning shedding light on etiology. Neuropsychologia, 43, 930-942.

Cropley, V. L., Fujita, M., Innis, R. B., \& Nathan, P. J. (2006). Molecular imaging of the dopaminergic system and its association with human cognitive function. Biological Psychiatry, 59, 898-907.

Crow, T. J. (1980). Positive and negative schizophrenic symptoms and the role of dopamine. The British Journal of Psychiatry: The Journal of Mental Science, 137, 383-386.

Davis, K. L., Kahn, R. S., Ko, G., \& Davidson, M. (1991). Dopamine in schizophrenia: A review and reconceptualization. The American Journal of Psychiatry, 148, 1474-1486.

Delis, D. C., Kramer, J. H., Kaplan, E., \& Ober, B. A. (2000). California Verbal Learning Test-Second Edition. New York: Psychological Corporation.

Erhart, S. M., Marder, S. R., \& Carpenter, W. T. (2006). Treatment of schizophrenia negative symptoms: Future prospects. Schizophrenia Bulletin, 32, 234-237. 
Erixon-Lindroth, N., Farde, L., Wahlin, T. B., Sovago, J., Halldin, C., \& Backman, L. (2005). The role of the striatal dopamine transporter in cognitive aging. Psychiatry Research, 138, 1-12.

Fried, I., Wilson, C. L., Morrow, J. W., Cameron, K. A., Behnke, E. D., \& Ackerson, L. C., et al. (2001). Increased dopamine release in the human amygdala during performance of cognitive tasks. Nature Neuroscience, 4, 201-206.

Galeno, R., Molina, M., Guirao, M., \& Isoardi, R. (2004). Severity of negative symptoms in schizophrenia correlated to hyperactivity of the left globus pallidus and the right claustrum: A PET study. The World Journal of Biological Psychiatry, 5, 20-25.

Gladsjo, J. A., McAdams, L. A., Palmer, B. W., Moore, D. J., Jeste, D. V., $\&$ Heaton, R. K. (2004). A six-factor model of cognition in schizophrenia and related psychotic disorders: Relationships with clinical symptoms and functional capacity. Schizophrenia Bulletin, 30, 739-754.

Goldberg, T. E., Egan, M. F., Gscheidle, T., Coppola, R., Weickert, T., Kolachana, B. S., et al. (2003). Executive subprocesses in working memory: Relationship to catechol-O-methyltransferase Val158Met genotype and schizophrenia. Archives of General Psychiatry, 60, 889896.

Goldman-Rakic, P. S. (2004). Targeting the dopamine D-1 receptor in schizophrenia: Insights for cognitive dysfunction. Psychopharmacology, 174, 3-16.

Goldman-Rakic, P. S., \& Selemon, L. D. (1997). Functional and anatomical aspects of prefrontal pathology in schizophrenia. Schizophrenia Bulletin, 23, 437-458.

Gottesman, I. I., \& Gould, T. D. (2003). The endophenotype concept in psychiatry: Etymology and strategic intentions. The American Journal of Psychiatry, 160, 636-645.

Green, M. F. (1996). What are the functional consequences of neurocognitive deficits in schizophrenia? The American Journal of Psychiatry, 153, 321-330.

Green, M. F., Kern, R. S., Braff, D. L., \& Mintz, J. (2000). Neurocognitive deficits and functional outcome in schizophrenia: Are we measuring the "right stuff"? Schizophrenia Bulletin, 26, 119-136.

Green, M. F., Kern, R. S., \& Heaton, R. K. (2004). Longitudinal studies of cognition and functional outcome in schizophrenia: Implications for MATRICS. Schizophrenia Research, 72, 41-51.

Green, M. F., Satz, P., Ganzell, S., \& Vaclav, J. F. (1992). Wisconsin Card Sorting Test performance in schizophrenia: Remediation of a stubborn deficit. The American Journal of Psychiatry, 149, 62-67.

Heaton, R. K. (1981). The Wisconsin Card Sorting Test. Odessa, FL: Psychological Assessment Resources.

Heinrichs, R. W., \& Zakzanis, K. K. (1998). Neurocognitive deficit in schizophrenia: A quantitative review of the evidence. Neuropsychology, 12, 426-445.

Heinz, A., Knable, M. B., Coppola, R., Gorey, J. G., Jones, D. W., Lee, K. S., et al. (1998). Psychomotor slowing, negative symptoms and dopamine receptor availability-An IBZM SPECT study in neuroleptictreated and drug-free schizophrenic patients. Schizophrenia Research, 31, 19-26.

Hellman, S. G., Kern, R. S., Neilson, L. M., \& Green, M. F. (1998). Monetary reinforcement and Wisconsin Card Sorting performance in schizophrenia: Why show me the money? Schizophrenia Research, 34, $67-75$.

Heydebrand, G., Weiser, M., Rabinowitz, J., Hoff, A. L., DeLisi, L. E., \& Csernansky, J. G. (2004). Correlates of cognitive deficits in first episode schizophrenia. Schizophrenia Research, 68, 1-9.

Juckel, G., Schlagenhauf, F., Koslowski, M., Filonov, D., Wüstenberg, T., Villringer, A., et al. (2006). Dysfunction of ventral striatal reward prediction in schizophrenic patients treated with typical, not atypical, neuroleptics. Psychopharmacology, 187, 222-228.

Juckel, G., Schlagenhauf, F., Koslowski, M., Wüstenberg, T., Villringer, A., Knutson, B., et al. (2006). Dysfunction of ventral striatal reward prediction in schizophrenia. NeuroImage, 29, 409.
Kaufman, A., \& Kaufman, N. (1990). Kaufman Brief Intelligence Test Manual. Circle Pines, MN: American Guidance Service.

Kimberg, D. Y., D'Esposito, M., \& Farah, M. J. (1997). Effects of bromocriptine on human subjects depend on working memory capacity. Neuroreport, 8, 3581-3585.

Kurtz, M. M., Moberg, P. J., Gur, R. C., \& Gur, R. E. (2001). Approaches to cognitive remediation of neuropsychological deficits in schizophrenia: A review and meta-analysis. Neuropsychology Review, 11, 197-210.

Lezak, M. D., Howieson, D. B., Loring, D. W., Hannay, H. J., \& Fischer, J. S. (2004). Neuropsychological assessment (4th ed.). New York: Oxford University Press.

Liddle, P. F. (1987). Schizophrenic syndromes, cognitive performance and neurological dysfunction. Psychological Medicine, 17, 49-57.

Liddle, P. F., Ngan, E. T., Duffield, G., Kho, K., \& Warren, A. J. (2002). Signs and Symptoms of Psychotic Illness (SSPI): A rating scale. The British Journal of Psychiatry: The Journal of Mental Science, 180, 45-50.

Lisman, J. E., \& Grace, A. A. (2005). The hippocampal-VTA loop: Controlling the entry of information into long-term memory. Neuron, 46, 703-713.

Mattay, V. S., Callicott, J. H., Bertolino, A., Heaton, I., Frank, J. A., Coppola, R., et al. (2000). Effects of dextroamphetamine on cognitive performance and cortical activation. NeuroImage, 12, 268-275.

Mehta, M. A., \& Rimel, R. W. (2006). Dopaminergic enhancement of cognitive function. Current Pharmaceutical Design, 12, 2487-2500.

Meyer-Lindenberg, A., Miletich, R. S., Kohn, P. D., Esposito, G., Carson, R. E., Quarantelli, M., et al. (2002). Reduced prefrontal activity predicts exaggerated striatal dopaminergic function in schizophrenia. Nature Neuroscience, 5, 267-271.

Milner, B. (1963). Effects of different brain lesions on card sorting-Role of the frontal lobes. Archives of Neurology, 9, 90-100.

Minzenberg, M. J., Poole, J. H., Benton, C., \& Vinogradov, S. (2004). Association of anticholinergic load with impairment of complex attention and memory in schizophrenia. The American Journal of Psychiatry, 161, 116-124.

Monchi, O., Petrides, M., Petre, V., Worsley, K., \& Dagher, A. (2001). Wisconsin Card Sorting revisited: Distinct neural circuits participating in different stages of the task identified by event-related functional magnetic resonance imaging. Journal of Neuroscience, 21, 7733-7741.

Moritz, S., Krausz, M., Gottwalz, E., Lambert, M., Perro, C., Ganzer, S., et al. (2000). Cognitive dysfunction at baseline predicts symptomatic 1-year outcome in first-episode schizophrenics. Psychopathology, 33, $48-51$.

Mozley, L. H., Gur, R. C., Mozley, P. D., \& Gur, R. E. (2001). Striatal dopamine transporters and cognitive functioning in healthy men and women. The American Journal of Psychiatry, 158, 1492-1499.

Nieoullon, A., \& Coquerel, A. (2003). Dopamine: A key regulator to adapt action, emotion, motivation and cognition. Current Opinion in Neurology, 16(Suppl 2), S3-S9.

O’Leary, D. S., Flaum, M., Kesler, M. L., Flashman, L. A., Arndt, S., \& Andreasen, N. C. (2000). Cognitive correlates of the negative, disorganized, and psychotic symptom dimensions of schizophrenia. The Journal of Neuropsychiatry and Clinical Neurosciences, 12, 4-15.

Owen, A. M., McMillan, K. M., Laird, A. R., \& Bullmore, E. (2005). N-back working memory paradigm: A meta-analysis of normative functional neuroimaging studies. Human Brain Mapping, 25, 46-59.

Pantelis, C., Harvey, C. A., Plant, G., Fossey, E., Maruff, P., Stuart, G. W., et al. (2004). Relationship of behavioural and symptomatic syndromes in schizophrenia to spatial working memory and attentional set-shifting ability. Psychological Medicine, 34, 693-703.

Potkin, S. G., Alva, G., Fleming, K., Anand, R., Keator, D., Carreon, D., et al. (2002). A PET study of the pathophysiology of negative symptoms in schizophrenia. Positron emission tomography. The American Journal of Psychiatry, 159, 227-237.

Resnick, S. M. (1992). Matching for education in studies of schizophrenia. Archives of General Psychiatry, 49, 246. 
Revheim, N., Schechter, I., Kim, D., Silipo, G., Allingham, B., Butler, P., et al. (2006). Neurocognitive and symptom correlates of daily problemsolving skills in schizophrenia. Schizophrenia Research, 83, 237-245.

Robbins, T. W. (2005). Chemistry of the mind: Neurochemical modulation of prefrontal cortical function. The Journal of Comparative Neurology, 493, 140-146.

Rund, B. R., Melle, I., Friis, S., Larsen, T. K., Midbøe, L. J., Opjordsmoen, S., et al. (2004). Neurocognitive dysfunction in first-episode psychosis: Correlates with symptoms, premorbid adjustment, and duration of untreated psychosis. The American Journal of Psychiatry, 161, 466-472.

Silver, H., Feldman, P., Bilker, W., \& Gur, R. C. (2003). Working memory deficit as a core neuropsychological dysfunction in schizophrenia. The American Journal of Psychiatry, 160, 1809-1816.

Snitz, B. E., MacDonald III, A. W., \& Carter, C. S. (2006). Cognitive deficits in unaffected first-degree relatives of schizophrenia patients: A meta-analytic review of putative endophenotypes. Schizophrenia Bulletin, 32, 179-194.

Sponheim, S. R., Steele, V. R., \& McGuire, K. A. (2004). Verbal memory processes in schizophrenia patients and biological relatives of schizophrenia patients: Intact implicit memory, impaired explicit recollection. Schizophrenia Research, 71, 339-348.

Stuss, D. T., Levine, B., Alexander, M. P., Hong, J., Palumbo, C., Hamer, L., et al. (2000). Wisconsin Card Sorting Test performance in patients with focal frontal and posterior brain damage: Effects of lesion location and test structure on separable cognitive processes. Neuropsychologia, 38, 388-402.

Summerfelt, A. T., Alphs, L. D., Wagman, A. M. I., Funderburk, F. R., Hierholzer, R. M., \& Strauss, M. E. (1991). Reduction of perseverative errors in patients with schizophrenia using monetary feedback. Journal of Abnormal Psychology, 100, 613-616.

Swanson, C. L., Gur, R. C., Bilker, W., Petty, R. G., \& Gur, R. E. (1998). Premorbid educational attainment in schizophrenia: Association with symptoms, functioning, and neurobehavioral measures. Biological Psychiatry, 44, 739-747.

Tabachnick, B. G., \& Fidell, L. S. (2007). Using multivariate statistics (5th ed.). Boston: Allyn \& Bacon.

Tanaka, S. (2006). Dopaminergic control of working memory and its relevance to schizophrenia: A circuit dynamics perspective. Neuroscience, 139, 153-171.

Taylor, S. F., Welsh, R. C., Wager, T. D., Phan, K. L., Fitzgerald, K. D., \& Gehring, W. J. (2004). A functional neuroimaging study of motivation and executive function. Neurolmage, 21, 1045-1054.

Thornton, A. E., Van Snellenberg, J. X., Sepehry, A. A., \& Honer, W. G. (2006). The impact of atypical antipsychotic medications on long-term memory dysfunction in schizophrenia spectrum disorder: A quantitative review. Journal of Psychopharmacology, 20, 335-346.

Toulopoulou, T., Morris, R. G., Rabe-Hesketh, S., \& Murray, R. M. (2003). Selectivity of verbal memory deficit in schizophrenic patients and their relatives. American Journal of Medical Genetics Part B.
Neuropsychiatric Genetics: The Official Publication of the International Society of Psychiatric Genetics, 116, 1-7.

Toulopoulou, T., Rabe-Hesketh, S., King, H., Murray, R. M., \& Morris, R. G. (2003). Episodic memory in schizophrenic patients and their relatives. Schizophrenia Research, 63, 261-271.

Van Snellenberg, J. X., Torres, I. J., \& Thornton, A. E. (2006). Functional neuroimaging of working memory in schizophrenia: Task performance as a moderating variable. Neuropsychology, 20, 497-510.

Volkow, N. D., Gur, R. C., Wang, G. J., Fowler, J. S., Moberg, P. J., Ding, Y. S., et al. (1998). Association between decline in brain dopamine activity with age and cognitive and motor impairment in healthy individuals. The American Journal of Psychiatry, 155, 344-349.

Vollema, M. G., Geurtsen, G. J., \& van Voorst, A. J. (1995). Durable improvements in Wisconsin Card Sorting Test performance in schizophrenic patients. Schizophrenia Research, 16, 209-215.

Watanabe, M., Kodama, T., \& Hikosaka, K. (1997). Increase of extracellular dopamine in primate prefrontal cortex during a working memory task. Journal of Neurophysiology, 78, 2795-2798.

Wechsler, D. (1997a). Wechsler Adult Intelligence Scale-Third Edition. New York: Psychological Corporation.

Wechsler, D. (1997b). Wechsler Memory Scale—Third Edition. New York: Psychological Corporation.

Weinberger, D. R. (1987). Implications of normal brain development for the pathogenesis of schizophrenia. Archives of General Psychiatry, 44, $660-669$

Wilk, C. M., Gold, J. M., McMahon, R. P., Humber, K., Iannone, V. N., \& Buchanan, R. W. (2005). No, it is not possible to be schizophrenic yet neuropsychologically normal. Neuropsychology, 19, 778-786.

Williams, G. V., \& Castner, S. A. (2006). Under the curve: Critical issues for elucidating D1 receptor function in working memory. Neuroscience, 139, 263-276.

Williams, G. V., \& Goldman-Rakic, P. S. (1995). Modulation of memory fields by dopamine D1 receptors in prefrontal cortex. Nature, 376, 572-575.

Wittmann, B. C., Schott, B. H., Guderian, S., Frey, J. U., Heinze, H. J., \& Düzel, E. (2005). Reward-related fMRI activation of dopaminergic midbrain is associated with enhanced hippocampus-dependent long-term memory formation. Neuron, 45, 459-467.

Wittorf, A., Klingberg, S., \& Wiedemann, G. (2004). Secondary verbal memory: A potential endophenotype of schizophrenia. Journal of Psychiatric Research, 38, 601-612.

Woodward, T. S., Ruff, C. C., Thornton, A. E., Moritz, S., \& Liddle, P. F. (2003). Methodological considerations regarding the association of Stroop and verbal fluency performance with the symptoms of schizophrenia. Schizophrenia Research, 61, 207-214.

Woodward, T. S., Thornton, A. E., Ruff, C. C., Moritz, S., \& Liddle, P. F. (2004). Material-specific episodic memory associates of the psychomotor poverty syndrome in schizophrenia. Cognitive Neuropsychiatry, 9, 213-227. 
Appendix

Medication Treatments

\begin{tabular}{|c|c|c|c|c|}
\hline Medication name & $n$ & Dose range (mg/day) & $M$ & $(S D)$ \\
\hline \multicolumn{5}{|l|}{ Antipsychotic medications } \\
\hline Amisulpride $^{\mathrm{a}}$ & 1 & & 800.0 & \\
\hline Aripiprazole $\mathrm{b}^{\mathrm{b}, \mathrm{c}}$ & 1 & & 15.0 & \\
\hline Chlorpromazine $^{\mathrm{b}, \mathrm{d}}$ & 1 & & 300.0 & \\
\hline Clozapine $\mathrm{b}^{\mathrm{b}, \mathrm{d}}$ & 29 & $37.5-825.0$ & 433.2 & (196.6) \\
\hline Loxapine $^{\mathrm{b}, \mathrm{d}}$ & 1 & & 75.0 & \\
\hline Methotrimeprazine $^{\mathrm{a}}$ & 1 & & 25.0 & \\
\hline Olanzapine $\mathrm{e}^{\mathrm{b}, \mathrm{d}}$ & 13 & $5.0-50.0$ & 19.6 & $(11.5)$ \\
\hline Perphenazine $^{\mathrm{d}, \mathrm{e}}$ & 1 & & 12.0 & \\
\hline Pimozide ${ }^{\mathrm{b}, \mathrm{d}}$ & 1 & & 2.0 & \\
\hline Quetiapine $^{\mathrm{b}, \mathrm{d}}$ & 9 & $200.0-800.0$ & 572.2 & $(234.7)$ \\
\hline Risperidone $^{\mathrm{b}, \mathrm{d}}$ & 8 & $1.0-6.0$ & 4.3 & (1.8) \\
\hline \multicolumn{5}{|l|}{ Antidepressant medications ${ }^{\mathrm{f}}$} \\
\hline Amitriptyline $\mathrm{d}^{\mathrm{d}}$ & 1 & & 50.0 & \\
\hline Citalopram $^{\mathrm{c}}$ & 2 & $10.0 / 20.0$ & 15.0 & (7.1) \\
\hline Fluoxetine $^{c}$ & 2 & $10.0 / 50.0$ & 30.0 & (28.3) \\
\hline Imipramine $^{\mathrm{d}}$ & 1 & & 25.0 & \\
\hline Paroxetine $^{\mathrm{d}}$ & 1 & & 15.0 & \\
\hline Sertraline $\mathrm{d}^{\mathrm{d}}$ & 2 & $100.0 / 125.0$ & 112.5 & $(17.7)$ \\
\hline Venlafaxine $^{\mathrm{c}}$ & 3 & $150-450$ & 275.0 & $(156.1)$ \\
\hline \multicolumn{5}{|l|}{ Mood stabilizers $^{\mathrm{f}, \mathrm{g}}$} \\
\hline$\overline{\text { Divalproex }}$ & 3 & $1,250.0-1,650.0$ & $1,500.0$ & (217.9) \\
\hline Carbamazepine & 1 & & 400.0 & \\
\hline Gabapentin & 2 & $1,200.0 / 2,400.0$ & $1,800.0$ & $(848.5)$ \\
\hline Lamotrigine & 1 & & 200.0 & \\
\hline Lithium carbonate & 4 & $600.0-1,800.0$ & $1,350.0$ & $(519.6)$ \\
\hline Topiramate & 2 & $100.0 / 100.0$ & 100.0 & $(0.0)$ \\
\hline Valproic acid & 7 & $500.0-3,000.0$ & $1,392.9$ & $(864.3)$ \\
\hline \multicolumn{5}{|l|}{ Anxiolytic medications ${ }^{\mathrm{f}, \mathrm{g}}$} \\
\hline$\overline{\text { Buspirone }}$ & 1 & & 20.0 & \\
\hline Clonazepam & 10 & $0.5-2.0$ & 1.5 & $(0.5)$ \\
\hline Lorazepam & 6 & $1.0-4.0$ & 1.8 & (1.5) \\
\hline Oxazepam & 2 & $5.0 / 15.0$ & 10.0 & (7.1) \\
\hline \multicolumn{5}{|l|}{ Antiparkinsonian Medications $\mathrm{s}^{\mathrm{f}}$} \\
\hline Benztropine ${ }^{\mathrm{d}}$ & 4 & $2.0-4.0$ & 3.0 & $(1.2)$ \\
\hline Procyclidine ${ }^{\mathrm{h}}$ & 1 & $2.0-7.0$ & 10.0 & (1.2) \\
\hline Depot antipsychotics $^{\mathrm{a}}$ & $n$ & Dosage (mg) & \multicolumn{2}{|c|}{ Injection interval } \\
\hline Flupenthixol decanoate & 2 & $20.0 / 100.0$ & \multicolumn{2}{|c|}{2 weeks } \\
\hline Fluphenazine decanoate & 1 & 37.5 & \multicolumn{2}{|c|}{2 weeks } \\
\hline Haloperidol decanoate & 1 & 75.0 & \multicolumn{2}{|c|}{3 weeks } \\
\hline Zuclopenthixol decanoate & 1 & 200.0 & \multicolumn{2}{|c|}{2 weeks } \\
\hline
\end{tabular}

${ }^{a}$ Reliable receptor affinity data could not be determined to allow calculation of antidopaminergic or anticholinergic load; cases for which these compounds were utilized were excluded from relevant analyses.

${ }^{\mathrm{b}}$ Estimates of antidopaminergic load were based upon ki values.

${ }^{\mathrm{c}}$ Anticholinergic load for the pharmacology scale was estimated to be noncontributory according to ki values for muscarinic antagonism.

${ }^{\mathrm{d}}$ Pharmacological and clinical indices for anticholinergic load were estimated on the basis of Minzenberg et al. (2004).

${ }^{\text {e }}$ Dopamine receptor affinity could not be determined accurately; this case was excluded from the relevant analyses.

${ }^{\mathrm{f}}$ Dopamine receptor affinity was presumed to be noncontributory on the basis of the compound's classification.

g Anticholinergic load was presumed to be noncontributory on the basis of the compound's classification.

${ }^{\mathrm{h}}$ Estimates of anticholinergic load for the pharmacology scale were available, but this case was excluded because of an additional depot treatment.

Received September 25, 2006 